\title{
The effects of severely restricted visual experience on the perception of "identity"
}

\author{
A. B. Woodruff and M. L. Slövak \\ NORTHERN ILLINOIS UNIVERSITY
}

\begin{abstract}
Abstraet
This study was concerned with the role of visual experience on identity perception. Hooded rats were used, 10 reared in darkness and 10 normally reared. As adult animals they were compared on learning to discriminate vertical and horizontal striations. No difference in performance was found. These findings are contrary to those predicted by Hebb (1949) and in agreement with those found by Woodruff (1951). It is concluded that prolonged visual experience is not a necessary requirement for simple identity visual perception.
\end{abstract}

\section{Introduetion}

Hebb (1949) presented a basis for a "revision of perceptual theory" in which, among other things, he attempted to point out the respective roles of inherited behavioral tendencies and learning in relatively simple visual discrimination tasks. He utilized two concepts, "primitive unity" and "identity." Primitive unity referred "to that unity and segregation from the background which seems to be a direct product of the pattern of sensory excitation and the inherited characteristics of the nervous system on which it acts" (Hebb, 1949, p. 19). This was demonstrated by a study (Hebb, 1937) in which albino rats, reared in darkness, oriented themselves in the direction of a figure against a ground the first time they received any visual stimulation. Identity is defined as "referring to the properties of association inherent in perception" (Hebb, 1949, p. 26) and "is dependent on a prolonged experience" (p. 28). This concept was illustrated by another aspect of his above mentioned study (Hebb, 1937) in which white rats, reared in darkness, learned to discriminate between vertical and horizontal striations using a Lashley jumping apparatus. These data were compared with those of normally reared animals in a study by Lashley (1938). Hebb's dark reared Ss took six times as many trials to learn this discrimination. Hebb (1958) and Riesen (1961) pointed out that experience is fundamental to the perception of "identity."

Woodruff (1951) reared 24 hooded rats in complete darkness until they were 150 days old and compared their performance in learning a vertical-horizontal striations discrimination with a normally reared group of rats. The apparatus was a Lashley jumping box, modified by an elevated $\mathrm{Y}$-maze set up to the openings. A correction method of running was used and the criterion of learning was two consecutive blocks of at least 9 correct trials out of 10 . Contrary to the above prediction, no difference was found between the two groups.
The discriminanda used in these studies were the same, but they do show some methodological differences. (1) Hebb-Lashley used a jumping response and Woodruff a running response. (2) The Hebb-Lashley Ss were first taught a black-white discrimination before they were introduced to the striations, but Woodruff's were not. (3) Hebb-Lashley used a noncorrection method and Woodruff used a correction method. (4) Most important Woodruff's Ss were hooded rats, Hebb used albino rats, but Lashley used "mature rats, with fully pigmented eyes, from an inbred strain derived from crossing Wistar albino stock with trapped wild." Thus, a genetic factor was introduced.

The present study further investigated the possible effects of being reared in complete darkness from birth on identity visual perception.

\section{Subjects}

The Ss were hooded rats between the ages of 90 and 95 days at the time they were introduced to the perceptual-learning situation. The experimental group $(\mathrm{E}, \mathrm{N}=10)$ of animals were reared in complete darkness from birth until the experiment was completed, except for the time spent in the actual running in the maze. The control group $(\mathrm{C}, \mathrm{N}=10)$ animals were reared under normal colony conditions.

Genetic effects were reduced by using litters from litter-mate females mated to a common litter-mate male.

\section{Apparatus}

The apparatus was a modified Grice box painted a neutral gray. The dimensions of the maze were: starting box, $161 / 2 \times 3$ in; choice-point area, $83 / 4 \times 5$ 1/2 in; with two sides $5 \times 4$ in at 45 degree angles; and two $11 \times 4$ in goal chambers. The height of the maze was 4 in. The starting box had a wood cover and the rest of the maze was covered with hardware cloth. Two Christmas tree light sockets, with seven watt frosted bulbs, were inserted into aluminum shields and mounted 2 in above the targets. They illuminated the targets and goal chambers, but the entrance to the runway was shielded from direct light exposure. There was no indication that the illumination had the disturbing effects observed by Riesen \& Aarons (1959).

The overhead-hung targets were $3 \times 33 / 4 \times 3 / 16$ in and were made of clear plastic material. Black and white rertical or horizontal striations $1 / 2$ in wide were painted with high gloss paint directly on the plastic. The striations came within $1 / 2$ in of each side of the target and $1 / 8$ in from the bottom and the top. Equal areas of the back of the targets were painted black. 
The experiment was performed in a completely darkened room. The only exposure the animal had to light was during the actual running in the maze.

\section{Procedure}

Pre-training: Both groups were given five 1-min. trials in the maze two days before the targets were placed in position, with no lights or targets. The day before blank targets were placed in position, each $\mathrm{S}$ was given a 1-min. trial under low illumination.

Training: The Ss learned to discriminate vertical striations (positive) from horizontal striations (negative). The position of the discriminanda was randomly alternated in each block of 10 trials with never over two consecutive trials, in which the correct target was on the same side. The Ss were run in blocks of 10 trials, with the trials spaced at 8-min. intervals. The criterion for learning was two consecutive blocks of 10 trials with at least nine correct choices. An error was defined as the $\mathrm{S}$ having at least the front legs and shoulders entering into the incorrect goal chamber.

The Ss were run under the conditions of $22 \mathrm{hr}$. of food deprivation. The reinforcement for a correct response was eating from a Purina Dog-Chow pellet.

\section{Results}

The mean number of trials to reach criterion for the Es was 187, with an S.D. of 32.41, and for the Cs was 182, with an S.D. of 39,51 . A t-test does not allow the rejection of the null hypothesis at a significant level $(t=.304 ; d f=18 ; p=.70)$. The conclusion of this evaluation was that there was no significant difference between the rats reared in the dark and those reared in the colony in learning to discriminate striations. These findings are in contradiction to those predicted by Hebb (1949) and in agreement with those of Woodruff (1951).

\section{Discussion}

These data suggest that simple "identity" may be a "product of the pattern of sensory excitation and the inherited characteristics of the nervous system of which it acts," the same as primitive unity. Hebb (1949, pp. 81. 83) suggests the possibility of this.

It may be that "identity" and "primitive unity" do not have to be conceptualized as "discrete processes of form perception" but may be regarded as points on a continuum of a single process. The findings of Hebb (1937), Woodruff (1951), and the present study would seem to indicate at least two points on this continuum. Hebb's (1937) dark-reared rats oriented themselves and moved towards a figure if it could be distinguished from its ground (primitive unity). In this experiment and in Woodruff (1951) rats reared in the dark learned to perceive the striations and to discriminate between them as rapidly as rats reared in the colony. This task required a level of identity which is quite simple and was not hindered by the lack of prolonged visual experience. A possible third point on the continuum could be suggested by another phase of Woodruff's (1951) study when he had his experimental and control groups learn to discriminate a card of $\mathrm{O}^{\prime} \mathrm{s}$ from one of $\mathrm{X}^{\prime} \mathrm{s}$. In this discrimination the animals with the greater amount of visual experience learned in significantly fewer trials. This is a more complex level of "identity" that might require "prolonged visual experience:"

The Hebb (1937) - Lashley (1938) data would not fit on this suggested dimension. However, since this comparison is confounded by a possible genetic difference, specifically manifested by the difference in the pigmentation of the eyes, it would seem that the present data and that of Woodruff (1951) provide more solid empirical data.

\section{References}

HEBB, D. O. The innate organization of visual activity. I. Perception of figures by rats reared in total darkness. Ped. Sem. and J. genet. Psychol., 1937, 51, 101-128.

HEBB, D. O. The organization of behavior. New York: John Wiley and Sons, 1949.

HEBB, D. O. A textbook of psychology. Philadelphia: W. B. Saunders Company, 1958.

LASHLEY, K. S. The mechanism of vision: XV: Preliminary studies of the rat's capacity for detail vision. J. gen. Psychol., 1938, 18, 123-193.

RIESEN, A. H. Stimulation as a requirement for growth and function in behavioral development. In D. Fiske and S. Maddi (Eds.) Functions of varied experience, Homewood (Illinois): The Dorsey Press, 1961.

RIESEN, A. H., \& Aarons, L. Visual movement and intensity discrimination in cats after early deprivation of pattern vision. $J$. comp. physiol. Psychol., 1959, 52, 142-149.

WOODRUFF, A. B. The effect of severe restriction on learning and perception in the rat. Unpublished doctoral dissertation, The Ohio State University, 1951. 